\title{
The Effect of Antibacterial Monomer MDPB on the Growth of Organisms Associated with Root Caries
}

\author{
Kazushi YOSHIKAWA ${ }^{1}$, Douglas T CLARK², Susan R BRAILSFORD², David BEIGHTON ${ }^{2}$, \\ Timothy F WATSON ${ }^{3}$, Satoshi IMAZATO ${ }^{4}$ and Yasuko MOMOI ${ }^{5}$ \\ ${ }^{1}$ Department of Operative Dentistry, Osaka Dental University, 8-1 Kuzuhahanazono-cho, Hirakata-shi, Osaka 573-1121, Japan \\ ${ }^{2}$ Department of Microbiology, ${ }^{3}$ Division of Conservative Dentistry, Guy's, King's and St Thomas' Dental Institute, Guy's \\ Tower, London Bridge, London, SE1 9RT, England \\ ${ }^{4}$ Department of Restorative Dentistry and Endodontology, Osaka University Graduate School of Dentistry, 1-8 Yamadaoka, \\ Suita, Osaka 565-0871, Japan \\ ${ }^{5}$ Department of Operative Dentistry, Tsurumi University School of Dental Medicine, 2-1-3 Tsurumi, Tsurumi-ku, Yokohama \\ 230-8501, Japan \\ Corresponding author, Satoshi IMAZATO; E-mail: imazato@dent.osaka-u.ac.jp
}

Received December 15, 2006 /Accepted January 18, 2007

\begin{abstract}
MDPB, 12-methacryloyloxydodecylpyridinium bromide, was tested for its ability to inhibit the growth of organisms associated with active root caries lesions and to modify the growth characteristics of these organisms at sub-MICs. MICs and MBCs of MDPB for independent isolates $(\mathrm{n}=5)$ of the following taxa: Streptococcus mutans, Streptococcus oralis, Streptococcus salivarius, Actinomyces naeslundii, Actinomyces israelii, Actinomyces gerensceriae, Actinomyces odontolyticus, Lactobacillus spp., and Candida albicans were determined, and the effects at sub-MIC on microbial growth kinetics were assessed. All isolates were sensitive to inhibition by MDPB. The median MICs and MBCs of MDPB for these organisms were in the range of 3.13 to $25.0 \mu \mathrm{g} / \mathrm{ml}$ and 6.25 to $50.0 \mu \mathrm{g} / \mathrm{ml}$, respectively. As for the influence of $\mathrm{pH}$, inhibition was sensitive to acidic $\mathrm{pH}$. Even at sub-MICs, the growth of all strains, measured as cell yield and doubling time, was significantly reduced. Based on the results of this study, MDPB exhibited the potential to inhibit the growth of microbiota associated with active root caries lesions.
\end{abstract}

Keywords: MDPB, Root caries, Plaque bacteria

\section{INTRODUCTION}

In tandem with the rapid growth of a dentate elderly population, occurrence of root surface caries is also on the increase ${ }^{1)}$. Restorative treatments of root surface caries are challenging in view of the inherent difficulties. Lesions often extend to approximal or subgingival surfaces, and the adequate removal of lesions and placement of restorations are difficult. Therefore, treatment options that can arrest active lesions with less surgical intervention are certainly beneficial.

Previously, we have reported that the progression of root caries could be inhibited by the application of an antibacterial adhesive system which incorporated 12-methacryloyloxydodecylpyridinium bromide $(\mathrm{MDPB})^{2)}$ using an in vitro demineralization model with a Streptococcus mutans culture technique ${ }^{33}$. The effectiveness of this management method depends upon the combined factors of the antimicrobial activity of MDPB and the sealing of the underlying, demineralized dentin from the oral environment. First, unpolymerized MDPB in the primer solution penetrates deeply into the lesion to kill/inactivate the infecting bacteria). Subsequently, the lesion is sealed with resin to prevent further ingress of fermentable dietary carbohydrates.

Antibacterial monomer MDPB, a compound synthesized from quaternary ammonium, exhibits strong antibacterial activity against oral streptococci $^{5)}$ or obligate anaerobes ${ }^{6)}$. Thus, it is expected to be effective when applied to natural root caries. However, root lesions contain other taxa including Actinomyces spp. and Candida albicans, which are aciduric and acidogenic, and found in large numbers associated with infected dentin of active root caries lesions ${ }^{7.9}$. Further, the intrinsic antibacterial characteristics of MDPB against organisms related to root surface caries need to be determined, not only under standard conditions but also in an acidic $\mathrm{pH}$ environment which prevails in the infected dentin of active root caries lesions. In this study, the hypothesis that MDPB is effective against organisms clinically isolated from root lesions was tested by measuring the minimum inhibitory/bactericidal concentrations (MIC/MBC) and examining bacterial growth kinetics in the presence of MDPB.

\section{MATERIALS AND METHODS}

\section{Microbial isolates}

The isolates used in this study were recovered from infected dentin, exposed after the removal of superficial dental plaque, of active root caries lesions. They were characterized by their proximity to the gingival margin and their soft texture evident upon probing ${ }^{10)}$. 
Five independent isolates of each species were used, whereby each isolate was recovered from a lesion in a different subject. These taxa represented the predominant members of the microflora of active root caries, namely, Streptococcus mutans, Streptococcus oralis, Streptococcus salivarius, Actinomyces naeslundii, Actinomyces israelii, Actinomyces gerensceriae, Actinomyces odontolyticus, Lactobacillus spp., and Candida albicans ${ }^{7.9}$. Although the actual range of phenotypes of these taxa relevant to the caries process was not known, this collection of strains would provide a good indication of the sensitivity range likely to be exhibited by strains in vivo.

Microorganisms were grown in the appropriate atmosphere settings on suitable media for 24 hours. Streptococcus spp. were cultured on Columbia agar base (Oxoid Ltd., Hampshire, UK) supplemented with $5 \%$ (v/v) defribinated horse blood (TCS Biosciences Ltd., Buckingham, UK) and Actinomyces spp. on Fastidious anaerobe agar (Lab M, Lancashire, UK) supplemented with $5 \%$ defibrinated horse blood. Inoculated plates were incubated anaerobically (MK3 Anaerobic Workstation, Don Whitley Scientific Ltd., West Yorkshire, UK) in an atmosphere of $10 \% \mathrm{CO}_{2}$, $10 \% \mathrm{H}_{2}$, and $80 \% \mathrm{~N}_{2}$ at $37^{\circ} \mathrm{C}$ for $24-48$ hours. C. albicans isolates were cultured on Sabouraud dextrose agar (Oxoid Ltd.) and incubated aerobically for 24 hours. Lactobacilli were cultured anaerobically on Rogosa agar (Oxoid Ltd.) for 24-48 hours.

\section{MIC and MBC determinations}

The minimum inhibitory concentration (MIC) and minimum bactericidal concentration (MBC) of MDPB, as $\mu \mathrm{g}$ per $\mathrm{ml}$, for each species were determined according to NCCLS guidelines ${ }^{11-13)}$ for the broth dilution method.

MDPB was prepared as previously described ${ }^{14}$ and dissolved in sterile distilled water at $1 \mathrm{mg} / \mathrm{ml}$. Suspensions of bacteria were prepared in $2 \mathrm{ml}$ of sterile $0.85 \%$ saline to a McFarland standard of 0.5 . The bacterial suspensions were diluted $1 / 10$ in sterile saline to give a final concentration of approximately $5 \times 10^{5}$ colony forming units $(\mathrm{CFU}) / \mathrm{ml}$. C. albicans isolates were suspended in $2 \mathrm{ml}$ of $0.85 \%$ saline. Turbidity was adjusted to a MacFarland standard of 0.5 , and suspensions were diluted $1 / 1000$ prior to use in MIC determinations.

Tests were performed in duplicate in 96-well microtiter trays. Two-fold dilutions at $100 \mu \mathrm{l}$ of the MDPB solution were added to wells covering a concentration range of $1.56-100 \mu \mathrm{g} / \mathrm{ml}$ and to which $100 \mu 1$ of the bacterial or fungal suspensions were added. All Streptococcus spp. were inoculated in cation-adjusted (10 mg/ $\mathrm{Ca}^{2+}, 10 \mathrm{mg} / \mathrm{l} \mathrm{Mg}^{2+}$ ) MuellerHinton broth (Oxoid Ltd.) supplemented with $2 \%$ (v/v) lysed horse blood (TCS Biosciences Ltd.). RPMI 1640 with $0.165 \mathrm{M}$ MOPS (Angus Buffers and
Biochemicals, Niagara Falls, USA) adjusted to $\mathrm{pH} 7.0$ with $1.0 \mathrm{M} \mathrm{NaOH}$ was used for testing Candida spp. Antimicrobial testing of Actinomyces spp. was performed in Schaedler broth (Oxoid Ltd.). Inoculated microtiter trays were incubated aerobically at $35^{\circ} \mathrm{C}$ for 24 hours, except for the Actinomyces spp. which were incubated in an anaerobic cabinet in an atmosphere consisting initially of $5 \% \mathrm{H}_{2}, 10 \% \mathrm{CO}_{2}$, and $85 \% \mathrm{~N}_{2}$ at $37^{\circ} \mathrm{C}$ for 48 hours.

Following incubation, the microtiter trays were examined using a light box. The MIC of an organism corresponded to the lowest concentration of MDPB that completely inhibited growth. To determine the MBC of MDPB for each isolate, $5 \mu 1$ from each well for the entire concentration range to be examined was spotted onto the appropriate medium. Following incubation as described above, the plates were examined for growth. The MBC for each isolate was described as the lowest concentration which exhibited no growth in the subculture. Individual wells were also selected randomly for cell culture purity test and viable cell count. In all cases, the cultures were pure and viable counts were as expected.

\section{Sub-MIC effects}

The effect of MDPB at sub-MIC concentration on the growth kinetics (doubling time $(\mathrm{h})$ and maximum optical density (OD) at $620 \mathrm{~nm}$, Fig. 1) of all organisms was determined by growing each organism in Brain Heart Infusion (Oxoid Ltd.) supplemented with a range of monomer concentrations in microtiter plates at $37^{\circ} \mathrm{C}$ for 48 hours (iEMS; Labsystems, Life Sciences International, Hampshire, UK). Solutions of MDPB at $0,1,5,10,25,50 \mu \mathrm{g} / \mathrm{ml}$ were tested based on the results of MIC/MBC determinations. Readings were taken hourly to facilitate calculation of the growth kinetic parameters. All determinations for each organism with each monomer concentration were made in duplicate, and the mean of these values was subjected to analysis. Data were accepted only if the duplicate determinations were

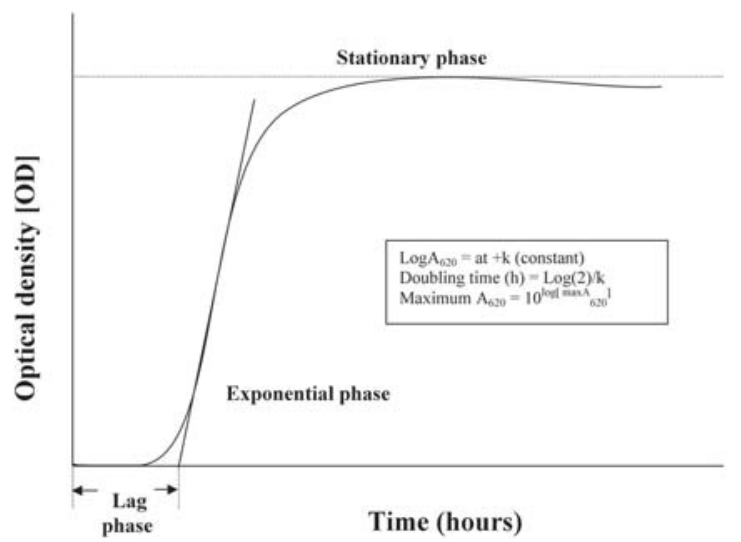

Fig. 1 Stylized growth curve showing phases of growth. 
identical, otherwise the strains were re-analyzed. This was an infrequent occurrence.

The effect of $\mathrm{pH}$ on the inhibitory activity of MDPB against the growth kinetics of Lactobacillus spp. and C. albicans was determined using the method described above. The $\mathrm{pH}$ values of the media were adjusted to 4.5, 5.0, 5.5, and 7.0 with $\mathrm{HCl}$ using a microelectrode (Microelectrodes Inc., Bedford, USA). The Actinomyces spp. and streptococci were not tested at the different $\mathrm{pH}$ values since preliminary experiments had shown that they grew poorly, if at all, at $\mathrm{pH} 5.0$ and 4.5 .

\section{Data analysis}

Growth of bacteria and yeasts in liquid medium can be considered to occur in phases (Fig. 1) - namely lag, exponential, and stationary phases. Each growth assay was set up in duplicate so that the mean doubling time and maximum OD were calculated from each growth curve. From these data, the mean doubling time and maximum growth rate were calculated for each species included in this study.

\section{RESULTS}

$M I C$ and $M B C$

Table 1 summarizes the MIC and MBC data. In most cases, except for A. naeslundii and C. albicans, the range of MIC values for each organism was within one dilution of the median, while the other two species showed a wide range of sensitivities. This heterogeneity was also reflected in the MBC determinations where the strains of these two species and S. salivarius isolated exhibited a wide range of values. For the other species, the MBCs were identical for all strains or within one dilution of the median value.

Table 1 MIC and MBC ( $\mu \mathrm{g} / \mathrm{ml})$ of MDPB for organisms isolated from active root caries lesions

\begin{tabular}{llll}
\hline \multicolumn{1}{c}{ Organism } & \multicolumn{2}{c}{ MIC } & \multicolumn{2}{c}{ MBC } \\
& Median (range) & \multicolumn{2}{c}{ Median (range) } \\
\hline S. mutans & $12.5(12.5)$ & 50.0 & $(50.0)$ \\
S. oralis & $12.5(12.5)$ & 25.0 & $(25.0-50.0)$ \\
S. salivarius & $12.5(6.25-12.5)$ & 50.0 & $(12.5-50.0)$ \\
A. naeslundii & $25.0(3.13-25.0)$ & 50.0 & $(6.25-50.0)$ \\
A. israelii & $12.5(12.5)$ & $25.0(25.0)$ \\
A. gerencseriae & $3.13(3.13)$ & $6.25(6.25)$ \\
A. odontolyticus & $6.25(6.25)$ & $12.5(12.5)$ \\
Lactobacillus spp. & $3.13(3.13-6.25)$ & $6.25(3.13-6.25)$ \\
C. albicans & $6.25(3.13-12.5)$ & $6.25(3.13-12.5)$ \\
\hline
\end{tabular}

Five independent isolates of each species were studied. Initial $\mathrm{pH}$ of medium was 7.4.
Effects of sub-MIC on growth

For all organisms, except for $S$. oralis, the presence of the lowest concentration of MDPB in the culture medium resulted in a reduction in the maximum OD achieved (Fig. 2A). S. oralis strains grew without inhibition in the medium supplemented with $5 \mu \mathrm{g} / \mathrm{ml}$ MDPB, and even with $10 \mu \mathrm{g} / \mathrm{ml}$ MDPB the extent of inhibition was less than that observed with any of the other species. The greater resistance of $S$. oralis to inhibition by the monomer was apparent in consideration of the changes in doubling time resulting from increasing MDPB concentrations (Fig. 2B).

\section{Effect of acidic environment on MDPB activity}

The effects of reduced $\mathrm{pH}$ on the growth of the Lactobacillus spp. and C. albicans strains are shown in Fig. 3. Reduction of $\mathrm{pH}$ in the culture medium from 7.0 to 閏 5.5 resulted in higher final maximum OD over the entire MDPB concentration range tested, up to $50 \mu \mathrm{g} / \mathrm{ml}$. The concentration of $50 \mu \mathrm{g} / \mathrm{ml} \mathrm{did}$ not completely inhibit the growth of Lactobacillus spp. tested here. The situation was similar for the five $C$. albicans strains tested, although not as pronounced. It was found that with MDPB at 50 $\mu \mathrm{g} / \mathrm{ml}$, growth was still present in media at $\mathrm{pH} 4.5$ and 5.0, but not at pH 5.5 and 7.0. With MDPB at $25 \mu \mathrm{g} / \mathrm{ml}$, growth of the C. albicans strains was completely inhibited at $\mathrm{pH} 7.0$ - but in media at $\mathrm{pH}$ 閏 5.5, growth was only around $50 \%$ inhibited. At the lowest $\mathrm{pH}$ used (i.e., $\mathrm{pH} 4.5$ ), inhibition of growth

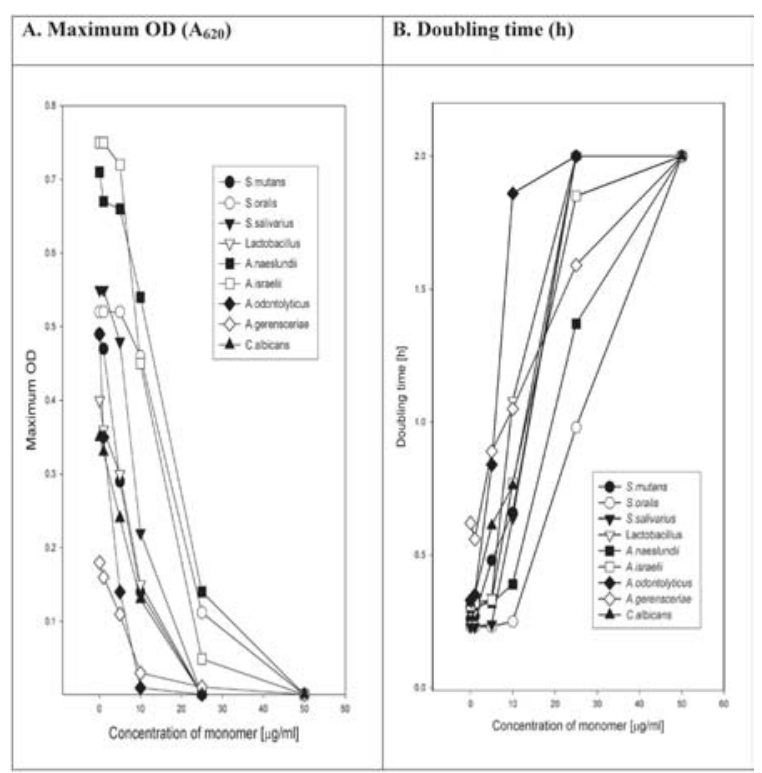

Fig. 2 Comparison of the maximum OD (A) and doubling time (B) achieved by each of the taxa included in this study over a range of MDPB concentrations. Values are means, and each point represents the mean of duplicate determinations for each of five isolates. 


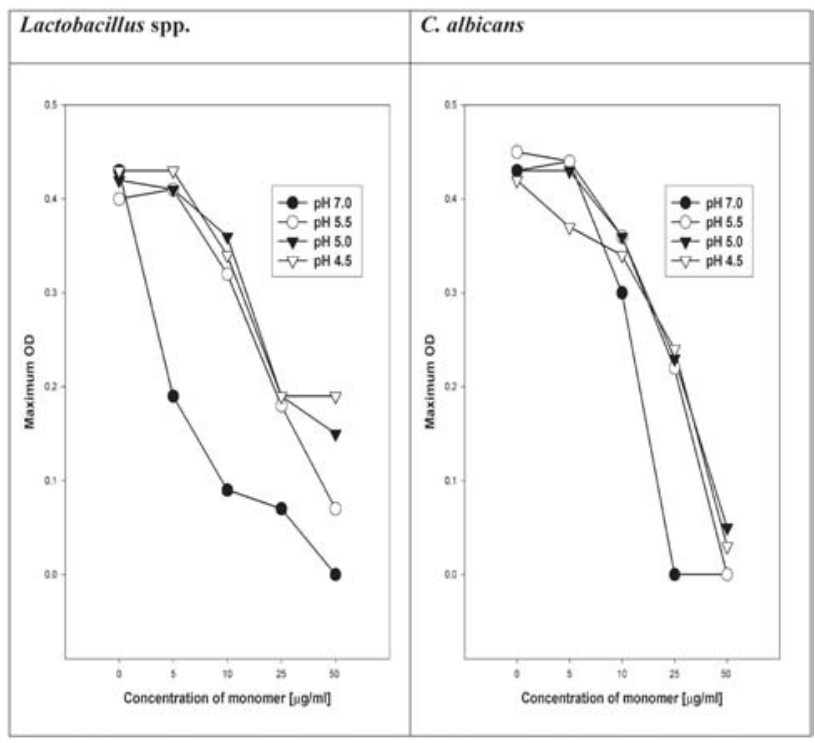

Fig. 3 Effects of medium $\mathrm{pH}$ on the maximum OD achieved by Lactobacillus spp. and C. albicans. Values are the mean of duplicate determinations for each of five strains.

was observed for C. albicans with only $5 \mu \mathrm{g} / \mathrm{ml}$ of MDPB.

\section{DISCUSSION}

The MICs and MBCs of MDPB for a range of oral microorganisms including oral streptococci ( $S$. mutans, S. sobrinus, $S$. oralis, $S$. salivarius, $S$. sanguis, $S$. gordonii), lactobacilli ( $L$. plantarum, $L$. salivarius, L. acidophilus, L. paracasei, L. brevis, $L$. salivarius ssp. salicinus), and a number of anaerobic bacteria (Propionibacterium acnes, Eubacterium alactolyticum, Bifidobacterium bifidum, and Peptostreptococcus asacchrolyticus) have previously been reported ${ }^{5,6)}$. In these studies, ${ }^{5,6)}$, only one strain of each species was tested. Nonetheless, these studies demonstrated that the streptococci had MICs in the range of 7.8 to $25.0 \mu \mathrm{g} / \mathrm{ml}$, while the lactobacilli and anaerobic rods had MICs in the range of 3.9 to 31.3 $\mu \mathrm{g} / \mathrm{ml}$. The different ranges undoubtedly arose from the use of different stock concentrations of MDPB in the MIC determination. The two mutans streptococcal strains tested were apparently the most susceptible to inhibition by MDPB, although they - amongst the streptococci - exhibited the highest MBCs.

In the present study, five independent isolates of each species were tested. The MICs for the oral streptococcal species tested were virtually identical, and they were within the same general range reported previously for this group of oral bacteria. However, S. mutans was not shown to be more susceptible to inhibition by MDPB than the other oral streptococci. Moreover, it seemed that the MBC of
MDPB for $S$. mutans was greater than those for the other streptococci, in agreement with the findings of a previous report ${ }^{5}$. The Lactobacillus spp. tested here had the lowest MIC and MBC, whereas previously members of this species had been reported to have higher MICs and much greater $\mathrm{MBCs}^{6)}$. Differences in results might be due to the media employed, and the compositions of culture media must be standardized in order to obtain reproducible and comparable results between laboratories ${ }^{11-13)}$. Nonetheless, the present data clearly demonstrated that MDPB was inhibitory to lactobacilli isolated directly from root caries lesions.

The MICs of MDPB for Actinomyces spp. and C. albicans have not been reported thus far, although these organisms are regularly isolated from active root caries lesions. The actinomyces exhibited a range of MICs from 3.13 to $25.0 \mu \mathrm{g} / \mathrm{ml}$ - with $A$. naeslundii being the most resistant and $A$. gerencseriae the most susceptible. The MBCs were one dilution higher than the MICs. As for the five C. albicans isolates, the MIC and MBC were the same, and this organism was very susceptible to inhibition by MDPB. On the overall, these data confirmed the previous observations ${ }^{5,6)}$ that MDPB in aqueous solution was inhibitory to the growth of oral bacteria at low concentrations, which should be achievable in vivo.

As for the effect of sub-MICs on growth, reduced growth yield and doubling time were clearly demonstrated. The mechanism by which MDPB inhibits growth is believed to be due to the cationic binding of the molecule to cell wall components, resulting in disturbed membrane function and ultimately leading to leakage of cytoplasmic components ${ }^{15}$. At sub-MICs of MDPB, bacteria have to expend energy maintaining their structural and functional integrity in the presence of MDPB. As a result, energy for growth is channeled into cellular repair.

Of interest was the finding that at acidic $\mathrm{pH}$, MDPB was not as active as at near neutral pH. This was probably a reflection of the chemistry of MDPB as a cationic detergent. It might be that the surfaces of the organisms, lactobacilli and candida, were modified such that they were less able to bind the antimicrobial at acidic $\mathrm{pH}$ than at around $\mathrm{pH}$ 7.0. Whatever was the basis for the inhibition of MDPB activity, this might have effects on the activity of $\mathrm{MDPB}$ in vivo. The $\mathrm{pH}$ of infected dentin associated with carious lesions is below 5.0, and in some instances it is below $4.0^{16}$. In these circumstances, the concentration of MDPB - either in solution or as a component of a dental material - required to inhibit the growth of organisms in dentin may have to be greater than previously considered.

However, the driving force behind acid production in lesions is dietary carbohydrates. With the 
placement of a filling, dietary sugars are eliminated and it may be that the host determines the $\mathrm{pH}$ under the restoration. Our recent findings on the nature of bacteria surviving under restorations suggested that the prevailing $\mathrm{pH}$ was not acidic, since aciduric organisms, lactobacilli and mutans streptococci, were not detectable and the predominant microbiota were associated with oral health and not disease ${ }^{17)}$. Nonetheless, such concerns may not be important, since it has been demonstrated that MDPB incorporated in a dentin primer of a commercial adhesive system (Clearfil Protect Bond; $\mathrm{pH}$ 2.0) showed significant bacterial killing within natural carious lesions ${ }^{18}$. Such a high level of microbicidal activity at low $\mathrm{pH}$ was perhaps due to the inclusion of $\mathrm{MDPB}$ at $5 \%$ (w/w) - equivalent to approximately $50 \mathrm{mg} / \mathrm{ml}$ in aqueous solution and much greater than the 50 $\mu \mathrm{g} / \mathrm{ml}$ used in the present study.

In conclusion, the median MICs and MBCs of MDPB for a range of organisms isolated from active root carious lesions were in the range of 3.13 to 25.0 $\mu \mathrm{g} / \mathrm{ml}$ and 6.25 to $50.0 \mu \mathrm{g} / \mathrm{ml}$, respectively. At subMIC levels, growth yields and rates were reduced thereby underlining the potential of MDPB for killing bacteria in vivo, although its inhibitory activity was reduced at acidic $\mathrm{pH}$.

\section{ACKNOWLEDGEMENTS}

This study was supported by a Grant-in-aid for Scientific Research (Nos. 15209066, 16390545, and 18659562) from the Japan Society for the Promotion of Science and the 21st Century COE at Osaka University Graduate School of Dentistry supported by the Ministry of Education, Culture, Sports, Science and Technology. This study was also supported in part by Kuraray Medical Co., Tokyo, Japan.

\section{REFERENCES}

1) Steele JG, Sheiham A, Marcenes W, Walls AWG. National diet and nutrition survey: people aged 65 years and over. Vol 2: Report of the oral health survey. London: Stationery Office, 1998.

2) Imazato S, Kinomoto $\mathrm{Y}$, Tarumi $\mathrm{H}$, Torii $\mathrm{M}$, Russell RRB, McCabe JF. Incorporation of antibacterial monomer MDPB into dentin primer. J Dent Res 1997; 76:768-772.

3) Kuramoto A, Imazato S, Walls AWG, Ebisu S. Inhibition of progression of root caries by an antibacterial adhesive. J Dent Res 2005; 84:89-93.

4) Imazato S, Walls AWG, Kuramoto A, Ebisu S. Penetration of an antibacterial dentine-bonding sys- tem into demineralized human root dentine in vitro. Eur J Oral Sci 2002; 110:168-174.

5) Imazato S, Ebi N, Tarumi H, Russell RRB, Kaneko T, Ebisu S. Bactericidal activity and cytotoxicity of antibacterial monomer MDPB. Biomaterials 1999; 20:899-903

6) Imazato S, Torii Y, Takatsuka T, Inoue K, Ebi N, Ebisu S. Bactericidal effect of dentin primer containing antibacterial monomer methacryloyloxydodecylpyridinium bromide (MDPB) against bacteria in human carious dentin. J Oral Rehabil 2001; 28:314319.

7) Brailsford SR, Tregaskis RB, Leftwich HS, Beighton D. The predominant Actinomyces spp. isolated from infected dentin of active root caries lesions. J Dent Res 1999; 78:1525-1534.

8) Brailsford SR, Shah B, Simons D, Gilbert S, Clark D, Ines I, Adams SE, Allison C, Beighton D. The predominant aciduric microflora of root-caries lesions. $\mathrm{J}$ Dent Res 2001; 80:1828-1833.

9) Beighton D, Lynch E. Relationships between yeasts and primary root-caries lesions. Gerodontology 1993; 10:105-108.

10) Beighton D, Lynch E, Heath MR. A microbiological study of primary root-caries lesions with different treatment needs. J Dent Res 1993; 72:623-629.

11) National Committee for Clinical Laboratory Standards. Methods for antimicrobial susceptibility testing of anaerobic bacteria, 5th ed, Approved standard. NCCLS publication M11-A5. National Committee for Clinical Laboratory Standards, Wayne, PA, 2001.

12) National Committee for Clinical Laboratory Standards. Method for dilution antimicrobial susceptibility testing for bacteria that grow aerobically, 5th ed, Approved standard. NCCLS publication M7-A5. National Committee for Clinical Laboratory Standards, Wayne, PA, 2000.

13) National Committee for Clinical Laboratory Standards. Reference method for broth dilution antifungal susceptibility testing of yeasts, Approved standard. NCCLS document M27-A2. National Committee for Clinical Laboratory Standards, Wayne, PA, 2002.

14) Imazato S, Russell RRB, McCabe JF. Antibacterial activity of MDPB polymer incorporated in dental resin. J Dent 1995; 23:177-181.

15) Scheie AA. Modes of action of currently known chemical anti-plaque agents other than chlorhexidine. J Dent Res 1989; 68:1609-1616.

16) Hojo S, Komatsu M, Okuda R, Takahashi N, Yamada T. Acid profiles and $\mathrm{pH}$ of carious dentin in active and arrested lesions. J Dent Res 1994; 73:1853-1857.

17) Paddick JS, Brailsford SR, Kidd EAM, Beighton D. Phenotypic and genotypic selection of microbiota surviving under dental restorations. Appl Environ Microbiol 2005; 71:2467-2472.

18) Smith G, Lumley PJ, Ruettermann S, Imazato S, Smith AJ. Antibacterial effects of a selfetching/priming system for composite resin restorations. J Dent Res 2003; 82:B343. 